\title{
Parálisis aislada del nervio hipogloso secundaria a quiste articular atlanto-occipital. Presentación de un caso y revisión de la literatura
}

\author{
J.M. Santín-Amo; D. Castro-Bouzas; A. Prieto-González; M. Arias-Gómez*; A. Arcos-Algaba; L. Díaz-Cabanas y \\ M. Gelabert-González
}

Servicios de Neurocirugía y Neurología*. Hospital Clínico Universitario de Santiago. Departamento de Cirugía. Universidad de Santiago de Compostela.

\section{Resumen}

La parálisis del nervio hipogloso se asocia comúnmente con la afectación de otros pares craneales. Su lesión aislada es infrecuente.

Presentamos el caso de un paciente en el cual la parálisis es debida a la presencia de un quiste yuxtafacetario a nivel de la articulación atlanto-occipital derecha. Revisamos la anatomía del nervio hipogloso, las diferentes opciones terapéuticas así como el diagnóstico diferencial y los trabajos publicados hasta la fecha.

PALABRAS CLAVE: Quiste yuxtafacetario atlantooccipital. Parálisis del nervio hipogloso. Anatomía del hipogloso.

Isolated hypoglossal nerve palsy secondary to an atlantoccipital joint synovial cyst. Case report and literature review

\section{Summary}

Hypoglossal nerve palsy is commonly associated with the involvement of other cranial nerves. His injury is rarely isolated. We present a patient in which paralysis is due to the presence of a "juxtafacet cyst" of the atlanto-occipital joint. We review the anatomy of the hypoglossal nerve, different therapeutic options, the differential diagnosis and papers published to date.

KEY WORDS: Atlanto-occipital juxtafacet cyst. Hypoglosal nerve palsy. Hypoglosal anatomy.

\section{Introducción}

La parálisis aislada del nervio hipogloso es una patología muy infrecuente; incluso aquella ocasionada por compresión a nivel de la porción cisternal del nervio podríamos

Recibido: 2-10-09. Aceptado: 12-01-10 calificarla como rara ${ }^{5}$. Muchas y diferentes patologías podrían afectar al nervio o a su vascularización en la cisterna prebulbar ocasionando la consecuente parálisis.

El término quiste yuxtafacetario ha sido empleado para referirse a aquellas lesiones quísticas que tienen su origen en el tejido conectivo periarticular de las facetas espinales. Estas lesiones son más frecuentes a nivel lumbar y su presencia en la unión atlanto-occipital es extremadamente $\operatorname{rara}^{6,7}$.

\section{Caso clínico}

Varón de 74 años sin clínica neurológica previa que presentó un cuadro clínico de dos meses de evolución que ocasionaba dificultad para hablar. Acude a urgencias del hospital donde tras la realización de una tomografía computarizada (TAC) craneal, informada como normal, es dado de alta. Ingresa para estudio y en la exploración física se comprueba que existe desviación lingual hacia el lado derecho y atrofia ipsilateral (Figura 1). Se practicó una resonancia magnética (RMN) cerebral con y sin gadolinio, que mostró la presencia de una lesión quística de $1 \mathrm{~cm}$ de

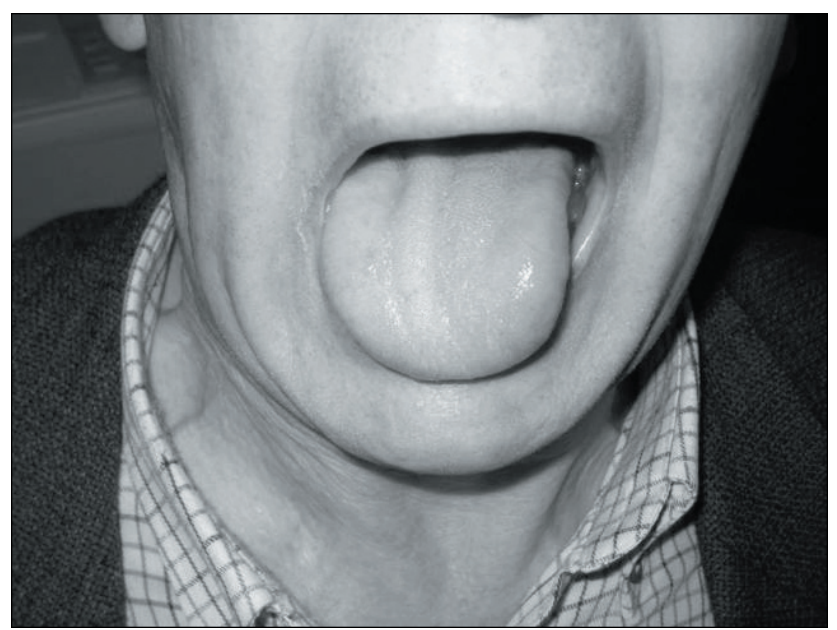

Figura1. Imagen del paciente en la cual es evidente la desviación de la lengua hacia el lado derecho y atrofia ipsilateral. 


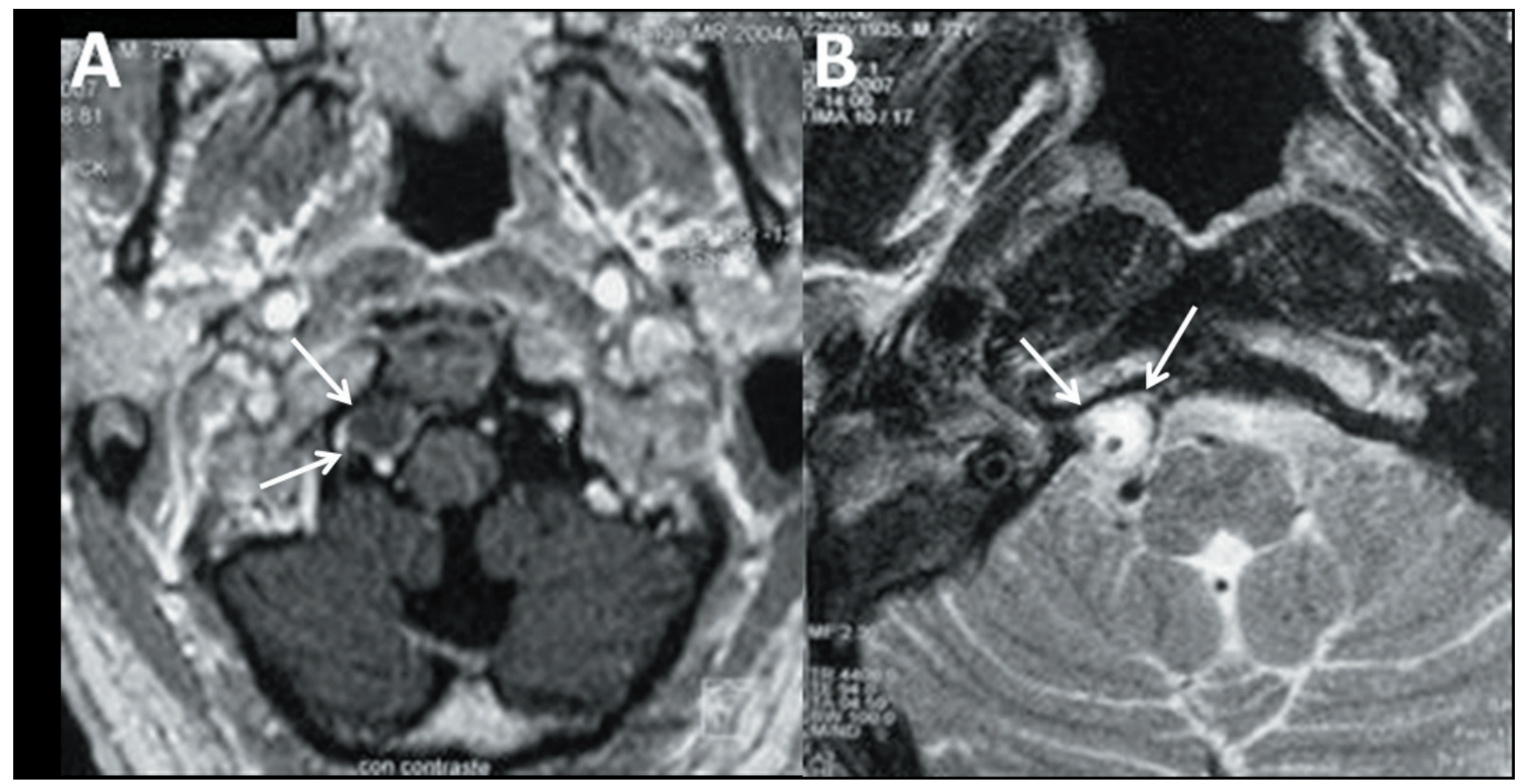

Figura 2. Cortes axiales de RMN en los que observamos la presencia de una lesión adyacente al agujero condíleo anterior derecho (flechas) isointensa en T1 (A) e hiperintensa en T2 (B).

diámetro en situación atlanto-occipital derecha (Figura 2). La lesión es isointensa en T1 e hiperintensa en T2 y no realza tras la administración de contraste. Se localiza adyacente al agujero condíleo anterior derecho y está asociada a alteraciones en la charnela cráneo-cervical, que sugieren patología inflamatoria y/o degenerativa articular. Comprime el nervio hipogloso en su trayecto cisternal.

El TAC de la unión cráneo-cervical muestra una lesión hipodensa asociada a cambios degenerativos a nivel de la articulación occípito-atloidea derecha.

Dado que el paciente, de edad avanzada, no presenta inestabilidad cervical, la parálisis del hipogloso está establecida y la situación clínica no se modifica decidimos tratamiento conservador y vigilancia radiológica. A los 12 meses del diagnóstico, la situación clínica permanecía inmodificable.

\section{Discusión}

El nervio hipogloso es puramente motor e inerva la musculatura intrínseca y extrínseca de la lengua. Su núcleo, formado por cuatro columnas diferenciadas, tiene una extensión bulbo-espinal, situándose en su porción bulbar en una posición paramediana, trígono del hipogloso, a nivel del suelo del IV ventrículo ${ }^{2}$. Las fibras nerviosas que originarán el nervio hipogloso, en número de 3 a 15 , abandonan el tronco entre el núcleo olivar inferior y la vía piramidal. En la cisterna medular las diferentes fibras se fusionan para dar lugar a un tronco común ${ }^{1}$. Recibe vascularización de las arterias medulares antero-laterales y laterales que pueden tener diferentes orígenes: arteria espinal anterior o posterior, ramas de la arteria ponto-medular (a su vez rama de la arteria basilar) o incluso la PICA.

El nervio tiene un trayecto cisternal dirigido inferior y antero-lateralmente. Las distintas raíces se dirigen hacia delante sobre la articulación atlanto-occipital para alcanzar el canal del hipogloso, emergiendo del mismo como un tronco común. El canal discurre hacia fuera y hacia delante a través del cóndilo occipital situado posteromedial e inferior al foramen yugular. Contiene además del nervio una rama meníngea de la arteria faríngea ascendente y un plexo de venas emisarias. Tras cursar entre la carótida y la yugular interna se distribuye a lo largo de la lengua ${ }^{8}$.

Las causas de una atrofia unilateral de la lengua son muy variadas y su origen puede ser central, medular o periférico. Thompson y Smoker ${ }^{13}$ describieron un esquema anatómico de la disfunción hipoglosa basándose en las diferentes patologías que podrían ocurrir a nivel medular, cisternal, base de cráneo, naso/orofaringe, espacio carotídeo y segmento sublingual. La parálisis aislada del nervio ocasionada por una compresión mecánica es un hecho extraordinariamente raro.

No hay grandes series en la literatura que describan parálisis aisladas del nervio hipogloso, lo habitual es que se asocie a lesiones de otras estructuras nerviosas como el glosofaríngeo, vago o espinal. Se han publicado diferentes 
Tabla 1

Sumario de los tres casos publicados previamente

\begin{tabular}{|c|c|c|c|}
\hline CARACTERÍSTICAS & Mujic et al $2003^{10}$ & Baldauf et al $2005^{3}$ & Elhammady et al $2009^{6}$ \\
\hline Edad & 52 & 51 & 67 \\
\hline Sexo & Varón & Mujer & Mujer \\
\hline Osteoartritis & Presente & No mencionada & Presente \\
\hline Síntomas & $\begin{array}{l}3 \text { semanas de disartria y } \\
\text { dificultad para deglutir }\end{array}$ & $\begin{array}{l}6 \text { meses de dificultad } \\
\text { para hablar }\end{array}$ & $\begin{array}{l}3 \text { meses de dolor facial } \\
\text { dificultad para hablar }\end{array}$ \\
\hline Características de RMN & $\begin{array}{l}\text { T1 Isointenso, sin realce } \\
\text { T2 Hiperintenso }\end{array}$ & $\begin{array}{l}\text { T1 Isointenso, sin realce } \\
\text { T2 Hiperintenso }\end{array}$ & $\begin{array}{l}\text { T1 Isointenso, sin realce } \\
\text { T2 Hiperintenso }\end{array}$ \\
\hline Abordaje & Farlateral & Farlateral & Juxtacondilar \\
\hline Evolución & $\begin{array}{l}2 \text { meses: persistencia } \\
\text { de la parálisis lingual }\end{array}$ & $\begin{array}{l}1 \text { año persistencia } \\
\text { de la parálisis lingual }\end{array}$ & $\begin{array}{l}1 \text { año persistencia } \\
\text { de la parálisis lingual }\end{array}$ \\
\hline
\end{tabular}

patologías como mecanismos causales de una parálisis aislada como fístulas durales ${ }^{4}$, schwannomas ${ }^{9}$, aneurismas de una arteria hipoglosa persistente ${ }^{1}$, fracturas del cóndilo occipital, cirugías del cuello o quistes aracnoideos ${ }^{11}$. En el trabajo de Keane ${ }^{9}$ donde revisa su experiencia personal con 100 casos de parálisis del hipogloso los tumores, malignos en su mayoría, representan el 50\%, siendo otras causas importantes los disparos con armas de fuego, los accidentes cerebrovasculares, esclerosis múltiple, la histeria o el síndrome de Guillain Barre.

Sin embargo la parálisis aislada del nervio hipogloso consecuencia de la existencia de un quiste sinovial es un hecho muy poco habitual, y solo aparece descrita en tres casos publicados en la literatura (Tabla 1)

La causa de los quistes sinoviales se desconoce, existiendo diferentes hipótesis. Para muchos su origen radica en una extrusión de la sinovial articular debido a un defecto capsular, otros creen que la etiología de los mismos se debe a una degeneración mixoide y no son pocos los que piensan que un traumatismo previo tendría mucho que ver con su formación ${ }^{6}$. Podrían ocurrir a cualquier nivel espinal, sin embargo son más prevalentes a nivel lumbar $(90 \%)$ que cervical $(5 \%)$ o torácico $(5 \%)^{14}$. Pueden causar inestabilidad vertebral, déficits neurológicos o cambios degenerativos. Los casos publicados rondan los 60 años y habitualmente presentan una historia de osteoartritis. Los principales síntomas son dificultades para el habla o deglución. La tomografía axial computarizada podría ayudar en su diagnóstico, pero la principal prueba diagnóstica es la $\mathrm{RMN}$ en la cual se presentan como lesiones isointensas en
T1 sin realce tras gadolinio e hiperintensas en T2. Debiendo diferenciarse de otras lesiones ocupantes de espacio de la misma localización como el schwannoma de nervio hipogloso, tumor del glomus o quistes epidermoides.

Aunque existe controversia en cuanto a su manejo, en todos los casos previamente descritos se opto por el tratamiento quirúrgico sin fusión espinal ${ }^{3,6,10}$. Sin embargo ninguno de los mismos recuperó la función del nervio hipogloso.

De acuerdo con Sabo et al. ${ }^{12}$ la fijación cervical debe ser considerada al tiempo de la cirugía resectiva si existen datos de inestabilidad prequirúrgica. El dolor postoperatorio en ausencia de fijación podría indicar inestabilidad de la articulación occípito-atloidea por lo que serían necesarias proyecciones en flexo-extensión para descartarla. La recidiva es rara a pesar de una escisión incompleta.

Nuestro paciente presentaba una lesión neurológica establecida y no presentaba signos clínicos ni radiográficos que hiciesen pensar en una inestabilidad cervical; se decidió una actitud conservadora, ya que en nuestra opinión y en ausencia de otra sintomatología, las posibilidades de recuperación de la parálisis del hipogloso eran escasas tal como ocurre con otras lesiones establecidas que afectan a los pares craneales.

\section{Conclusiones}

La parálisis aislada del nervio hipogloso ocasionada por la compresión del nervio en su trayecto cisternal debido a la presencia de un quiste de la articulación occipito-atloideo 
es extremadamente rara. Se trata de pacientes en la $5^{\mathrm{a}}-6^{\mathrm{a}}$ décadas de la vida con patología osteo-articular asociada y que debutan con dificultades para el habla y deglución. La prueba fundamental para su diagnóstico es la RMN. En cuanto a su tratamiento existe controversia optándose por la cirugía siempre que asocie inestabilidad occipito-cervical; la existencia de déficit neurológico es una indicación relativa para el tratamiento quirúrgico, ya que las posibilidades de recuperación del déficit neurológico tras la cirugía son escasas por lo que el tratamiento conservador parece también una buena opción.

\section{Bibliografía}

1. Al-Memar, A., Thrush, D.: Unilateral hypoglossal nerve palsy due to aneurysm of the stump of persistent hypoglossal artery. J Neurol Neurosurg Psychiatry 1998; 64: 405.

2. Bademci, G., Yaşargil, M.G.: Microsurgical anatomy of the hypoglossal nerve. J Clin Neurosc 2006; 13: 841-847.

3. Baldauf, J., Junghans, D., Schroeder, H.W.S.: Endoscope-assisted microsurgical resection of an intraneural ganglion cyst of the hypoglossal nerve. Case report. J Neurosurg 2005; 103: 920-922.

4. Blomquist, M.H., Barr, J.D., Hurst, R.W.: Isolated unilateral hypoglossal neuropathy caused by dural arteriovenous fistula. AJNR 1998; 19: 951-953.

5. Combarros, O., Alvarez de Arcaya, A., Berciano, J.: Isolated unilateral hypoglossal nerve palsy: nine cases. J Neurol 1998; 245: 98-100.

6. Elhammady, M.S., Farhat, H., Aziz-Sultan, M.A., Morcos, J.J.: Isolated unilateral hypoglossal nerve palsy secondary to an atlantooccipital joint juxtafacet synovial cyst. Case report and review of the literature. J Neurosurg Spine 2009; 10: 234-239.

7. Gelabert-González, M., Prieto-González, A., Santin-
Amo, J.M., Serramito-García R., García-Allut, A.: Lumbar synovial cyst in an adolescent: case report. Childs Nerv Syst 2009; 25: 719-721.

8. Karasu, A., Cansever, T., Batay, F., Sabanci, P.A., AlMefty, O.: The microsurgical anatomy of the hypoglossal canal. Surg Radiol Anat 2009; 31: 363-367.

9. Keane, J.R.: Twelfth-nerve palsy. Analysis of 100 cases. Arch Neurol 1996; 53: 561-566.

10. Mujic, A., Hunn, A., Liddell, J.,Taylor, B., Havlat, M., Beasley, T.: Isolated unilateral hypoglossal nerve paralysis caused by an atlanto-occipital joint synovial cyst. J Clin Neurosci. 2003; 10: 492-495.

11. Ryu, H., Makino, A., Hinokuma, K.: An arachnoid cyst involving only the hypoglossal nerve: case report and review of the literature. Br J Neurosurg 1999; 13: 492-495.

12. Sabo, R.A., Tracy, P.T., Weinger, J.M.: A series of 60 juxtafacet cysts: clinical presentation, the role of spinal instability, and treatment. J Neurosurg 1996; 85: 560-565.

13. Thompson, E.O., Smoker, W.R.: Hypoglossal nerve palsy: a segmental approach. Radiographics 1994; 14: 939-958.

14. Vaquero, J., Martínez, R., Aragonés, P., Piqueras, C.: Spinal synovial cyst with dural attachment. Neurocirugía 1992; 3: 35-37.

Santín-Amo, J.M.; Castro-Bouzas, D.; Prieto-González, A.; Arias Gómez, M.; Arcos- Algaba, A.; Díaz-Cabanas, L.; Gelabert-González, M.: Parálisis aislada del nervio hipogloso secundaria a quiste articular atlanto-occipital. Presentación de un caso y revisión de la literatura. Neurocirugía 2010; 21: 322-325.

Correspondencia: José María Santín Amo. Servicio de Neurocirugía. Hospital Clínico Universitario de Santiago de Compostela. A Choupana s/n. 15706 Santiago de Compostela

cochemelide@hotmail.com 